\title{
RF-chain ADC Resolution Trade-off in MIMO Hybrid Architecture
}

\author{
Satya Joshi and Markku Juntti \\ Centre for Wireless Communications, University of Oulu, P.O. Box 4500, FIN-90014, Oulu, Finland, \\ \{satya.joshi, markku.juntti\}@oulu.fi
}

\begin{abstract}
The problem of spectral efficiency maximization for hybrid analog/digital transceiver architecture, subject to both transmit and receiver power constraints, is considered. With the processing power constraint of the receiver, the number of radio frequency (RF) chains and analog-to-digital (ADC) resolution used greatly affect the achievable transmission rate. We formulate an optimization problem to find this trade-off, and provide an efficient algorithm to solve it. Numerical results show that for power constrained hybrid receiver, there is a trade-off between the number of RF chains and the ADC resolution, that can obtain maximal spectral efficiency.

Index Terms - Hybrid analog/digital beamforming, millimeter wave communication, analog-to-digital converters.
\end{abstract}

\section{INTRODUCTION}

There is growing interest in the exploration of underutilized millimeter wave (mm-wave) frequency spectrum for the future-generation wireless networks [1], because the available multi-GHz bandwidth in the mm-wave band is essential to meet very high peak data rate and low latency requirements of the next-generation wireless networks. Moreover, the small wave-length of mm-wave band signal allows the use of very large antenna arrays at both the transmitter and receiver, and it can provide high array gain, which is essential to compensate for the large path loss in the radio channel, and to provide large numbers of spatial degrees of freedom [2].

In conventional multiple-input multiple-output (MIMO) systems, precoder/combiner is generally designed at baseband via digital processors [3]. This fully-digital precoding/combining requires radio frequency $(\mathrm{RF})$ chains, including signal mixers, digital-to-analog/analog-to-digital converters (DAC/ADC), as many as the number of antenna elements. As mm-wave communication requires a large scale MIMO system to combat the propagation loss, the use of fully-digital precoding/combining techniques for mm-wave communication is not practically feasible, because of its huge implementation cost and power consumption. Given such constraints, the use of hybrid (ana$\log /$ digital beamforming) architecture with a low resolution $\mathrm{ADC} / \mathrm{DAC}$ is a potential solution [4], [5].

We consider the problem of spectral efficiency maximization for hybrid analog/digital transceiver architecture, subject

The research was supported by Academy of Finland 6Genesis Flagship (grant 318927), Finnish Funding Agency for Technology and Innovation (Tekes), Nokia, Esju, and CoreHW.
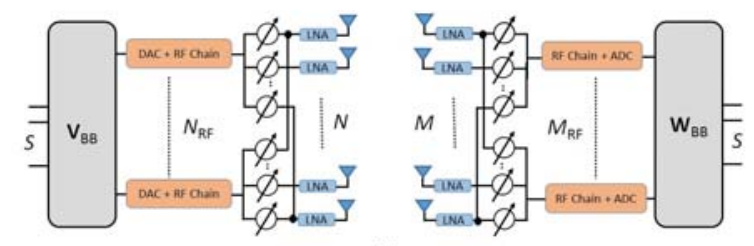

(a)
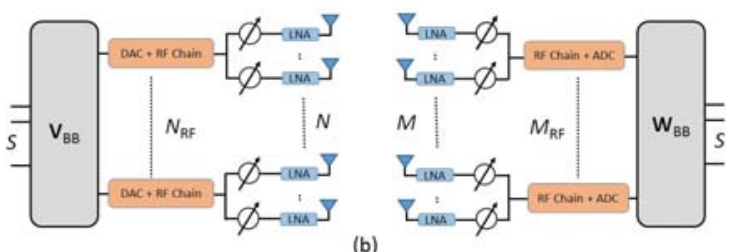

Fig. 1. Hybrid architectures: a) fully-connected, b) partially-connected.

to both transmit and receiver (processing) power constraints. With the power constraint of the receiver, there exist a tradeoff: whether to use low resolution ADC with a large number of antennas and RF chains, or to use high resolution ADC with few antennas and RF chains. The main contribution of the paper is to evaluate this trade-off, and propose an efficient algorithm to design hybrid analog/digital beamformers for mm-wave communication.

\section{System Model}

We consider a MIMO system with hybrid analog/digital architecture as shown in Fig. 1. The transmitter and receiver are equipped with $N$ and $M$ antennas, respectively. The set of transmit and receive antennas are denoted by $\mathcal{N}$ and $\mathcal{M}$, respectively. We denote the set of RF chains in the transmitter by $\mathcal{N}_{\mathrm{RF}}=\left\{1, \ldots, N_{\mathrm{RF}}\right\}$. The set of RF chains in the receiver is denoted by $\mathcal{M}_{\mathrm{RF}}=\left\{1, \ldots, M_{\mathrm{RF}}\right\}$. It is assumed that $N_{\mathrm{RF}} \leq N$ and $M_{\mathrm{RF}} \leq M$; and the transmitter and receiver communicate via $S$ data streams, with $S \leq \min \left(N_{\mathrm{RF}}, M_{\mathrm{RF}}\right)$.

We denote a set of antennas that are connected to $n$th RF chain in the transmitter by $\mathcal{N}(n)$, and its complement $\overline{\mathcal{N}}(n)=$ $\mathcal{N} \backslash \mathcal{N}(n)$ denotes the set of antennas that are not connected to $n$th RF chain. Similarly, the set of antennas that are connected to $m$ th RF chain in the receiver is denoted by $\mathcal{M}(m)$, and its complement is denoted by $\overline{\mathcal{M}}(m)=\mathcal{M} \backslash \mathcal{M}(n)$. In the case of fully-connected hybrid architecture (Fig. 1(a)), we have 


$$
\begin{aligned}
& R\left(\mathbf{V}, \mathbf{W}_{\mathrm{RF}}, \mathbf{W}_{\mathrm{BB}}, b\right)=B \log _{2}\left|\mathbf{I}_{S}+\left(1-\beta_{b}\right) \mathbf{X}^{-1} \mathbf{W}_{\mathrm{BB}}^{\mathrm{H}} \mathbf{W}_{\mathrm{RF}}^{\mathrm{H}} \mathbf{H V} \mathbf{V}^{\mathrm{H}} \mathbf{H}^{\mathrm{H}} \mathbf{W}_{\mathrm{RF}} \mathbf{W}_{\mathrm{BB}}\right|, \\
& \text { where, } \mathbf{X}=\sigma^{2}\left(1-\beta_{b}\right) \mathbf{W}_{\mathrm{BB}}^{\mathrm{H}} \mathbf{W}_{\mathrm{RF}}^{\mathrm{H}} \mathbf{W}_{\mathrm{RF}} \mathbf{W}_{\mathrm{BB}}+\beta_{b} \mathbf{W}_{\mathrm{BB}}^{\mathrm{H}} \mathrm{E}\left\{\operatorname{diag}\left(\mathbf{W}_{\mathrm{RF}}^{\mathrm{H}} \mathbf{y} \mathbf{y}^{\mathrm{H}} \mathbf{W}_{\mathrm{RF}}\right)\right\} \mathbf{W}_{\mathrm{BB}} .
\end{aligned}
$$

$|\mathcal{N}(n)|=N$ and $|\mathcal{M}(m)|=M$ for all $n \in \mathcal{N}_{\mathrm{RF}}, m \in \mathcal{M}_{\mathrm{RF}}$.

We denote $\mathbf{V}_{\mathrm{RF}} \in \mathbb{C}^{N \times N_{\mathrm{RF}}}$ as an analog precoder, and $\mathbf{V}_{\mathrm{BB}} \in \mathbb{C}^{N_{\mathrm{RF}} \times S}$ as a baseband digital precoder. Let $\mathbf{V}=$ $\mathbf{V}_{\mathrm{RF}} \mathbf{V}_{\mathrm{BB}}$, then the received signal can be expressed as

$$
\mathbf{y}=\mathbf{H V d}+\mathbf{z},
$$

where $\mathbf{H} \in \mathbb{C}^{M \times N}$ represents the channel matrix, $\mathbf{d} \in \mathbb{C}^{S}$ is a vector of data stream such that $\mathrm{E}\left\{\mathbf{d d}^{\mathrm{H}}\right\}=\mathbf{I}_{S}$, and $\mathbf{z} \sim$ $\mathcal{C N}\left(0, \sigma^{2} \mathbf{I}\right)$ is white Gaussian noise. We assume that each element of analog precoder $\mathbf{V}_{\mathrm{RF}}$ is implemented using a phase shifter, such that $\left|\mathbf{V}_{\mathrm{RF}}(k, n)\right|^{2}=1$ and $\mathbf{V}_{\mathrm{RF}}(\bar{k}, n)=0$ for all $k \in \mathcal{N}(n), \bar{k} \in \overline{\mathcal{N}}(n)$, and $n \in \mathcal{N}_{\mathrm{RF}}$.

Let $\mathbf{W}_{\mathrm{RF}} \in \mathbb{C}^{M \times M_{\mathrm{RF}}}$ and $\mathbf{W}_{\mathrm{BB}} \in \mathbb{C}^{M_{\mathrm{RF}} \times S}$ denote the analog and baseband digital combiner matrices, respectively. The estimated data vector $\hat{\mathbf{d}}$ obtained by analog/digital combining and ADC quantization can be written as

$$
\hat{\mathbf{d}}=\mathbf{W}_{\mathrm{BB}}^{\mathrm{H}} \mathcal{Q}\left(\mathbf{W}_{\mathrm{RF}}^{\mathrm{H}} \mathbf{y}\right)=\mathbf{W}_{\mathrm{BB}}^{\mathrm{H}} \mathcal{Q}\left(\mathbf{W}_{\mathrm{RF}}^{\mathrm{H}} \mathbf{H} \mathbf{x}+\mathbf{W}_{\mathrm{RF}}^{\mathrm{H}} \mathbf{z}\right),
$$

where $\mathcal{Q}(\cdot)$ denotes a scalar quantization function, which applies component-wise. Similar to $\mathbf{V}_{\mathrm{RF}}$, we assume that each element of matrix $\mathbf{W}_{\mathrm{RF}}$ is implemented using a phase shifter.

We apply additive quantization noise model [6] to decompose the quantized signal (2), and it can be reduced to [6]

$$
\hat{\mathbf{d}}=\mathbf{W}_{\mathrm{BB}}^{\mathrm{H}}\left\{\left(1-\beta_{b}\right)\left(\mathbf{W}_{\mathrm{RF}}^{\mathrm{H}} \mathbf{H} \mathbf{x}+\mathbf{W}_{\mathrm{RF}}^{\mathrm{H}} \mathbf{z}\right)+\mathbf{z}_{q}\right\},
$$

where $\beta_{b}$ is $b$-bit ADC distortion factor, and $\mathbf{z}_{q}$ is quantization noise with covariance $\beta_{b}\left(1-\beta_{b}\right) \mathrm{E}\left\{\operatorname{diag}\left(\mathbf{W}_{\mathrm{RF}}^{\mathrm{H}} \mathbf{y} \mathbf{y}^{\mathrm{H}} \mathbf{W}_{\mathrm{RF}}\right)\right\}$.

We assume a non-uniform quantizer, and in this case the distortion factor $\beta_{b}$ can be approximated as $\beta_{b} \approx \frac{\pi \sqrt{3}}{2} 2^{-2 b}$ for $b>3$ [6]. For $b \leq 3$ the value of $\beta_{b}$ is given in [7].

\section{A. Power Consumption Model of Receiver}

Let $P_{\mathrm{PS}}, P_{\mathrm{RF} \text { chain }}, P_{\mathrm{LNA}}, P_{\mathrm{ADC}}(b)$, and $P_{\mathrm{BB}}$ denote the power consumptions of a phase shifter (PS), RF chain, low noise amplifier (LNA), $b$-bit ADC, and baseband processor.

We assume that a PS can be turned on/off to save receiver power consumption. Let $a_{k m}$ be a binary variable associated with a PS connecting $k$ th antenna with $m$ th RF chain. Then total power consumption by the PSs becomes $P_{\mathrm{PS}} \sum_{m \in \mathcal{M}_{\mathrm{RF}}} \sum_{k \in \mathcal{M}(m)} a_{k m}$. Note that $m$ th $\mathrm{RF}$ chain is required to be enabled, if any PS connected to it is turned on. Hence, we define a binary variable $c_{m}=\max _{k \in \mathcal{M}(m)} a_{k m}$ associated with $m$ th RF chain. Therefore, the power consumption of the receiver can be approximated as

$$
\begin{aligned}
& P_{\mathrm{rx}}(\mathbf{a}, b, \mathbf{c}, \mathbf{e})=P_{\mathrm{PS}} \sum_{m \in \mathcal{M}_{\mathrm{RF}}} \sum_{k \in \mathcal{M}(m)} a_{k m}+\sum_{m \in \mathcal{M}_{\mathrm{RF}}} c_{m}\left(P_{\mathrm{RF} \text { chain }}\right. \\
&\left.+2 P_{\mathrm{ADC}}(b)\right)+\sum_{k \in \mathcal{M}} e_{k} P_{\mathrm{LNA}}+P_{\mathrm{BB}},
\end{aligned}
$$

where $e_{k}=\max _{m \in \mathcal{M}_{\mathrm{RF}}} a_{k m}$ is a variable associated with $k$ th receive antenna's LNA; $e_{k}$ is one, if any PS connected to $k$ th receive antenna is selected. Furthermore, we approximate $P_{\mathrm{ADC}}(b)=\xi f_{s} 2^{b}$, where $\xi$ is the ADC's energy consumption per conversion step and $f_{s}$ is the Nyquist sampling rate [8].

\section{B. Problem Formulation}

We consider a downlink scenario, where power and cost requirements in a base station (transmitter) is not stringent as compared with a mobile device (receiver). Therefore, we assume that the base station is equipped with high-resolution ADCs/DACs. Then, by assuming that the transmitter and receiver power allocations are subject to $\operatorname{tr}\left(\mathbf{V} \mathbf{V}^{\mathrm{H}}\right) \leq P_{\mathrm{tx}}^{\max }$ and $P_{\mathrm{rx}}(\mathbf{a}, b, \mathbf{c}, \mathbf{e}) \leq P_{\mathrm{rx}}^{\max }$, respectively, the problem of spectral efficiency maximization with the $b$-bit ADCs in the receiver can be expressed as

$$
\begin{array}{ll}
\text { maximize } & R\left(\mathbf{V}, \mathbf{W}_{\mathrm{RF}}, \mathbf{W}_{\mathrm{BB}}, b\right) \\
\text { subject to } & \operatorname{tr}\left(\mathbf{V} \mathbf{V}^{\mathrm{H}}\right) \leq P_{\mathrm{tx}}^{\max } \\
& \mathbf{V}=\mathbf{V}_{\mathrm{RF}} \mathbf{V}_{\mathrm{BB}} \\
& \left|\mathbf{V}_{\mathrm{RF}}(k, n)\right|^{2}=1, \quad k \in \mathcal{N}(n), n \in \mathcal{N}_{\mathrm{RF}} \\
& \mathbf{V}_{\mathrm{RF}}(k, n)=0, \quad k \in \overline{\mathcal{N}}(n), n \in \mathcal{N}_{\mathrm{RF}} \\
& P_{\mathrm{rx}}(\mathbf{a}, b, \mathbf{c}, \mathbf{e}) \leq P_{\mathrm{rx}}^{\max } \\
& \left|\mathbf{W}_{\mathrm{RF}}(k, m)\right|^{2}=a_{k m}, k \in \mathcal{M}, m \in \mathcal{M}_{\mathrm{RF}} \\
& \max _{k \in \mathcal{M}(m)} a_{k m}=c_{m}, \quad m \in \mathcal{M}_{\mathrm{RF}} \\
& \max _{m \in \mathcal{M}} a_{k m}=e_{k}, \quad k \in \mathcal{M} \\
& a_{k m}=\{0,1\}, \quad k \in \mathcal{M}(m), m \in \mathcal{M}_{\mathrm{RF}} \\
& c_{m}=\{0,1\}, \quad m \in \mathcal{M}_{\mathrm{RF}} \\
& e_{k}=\{0,1\}, \quad k \in \mathcal{M} \\
& a_{k m}=0, \quad k \in \overline{\mathcal{M}}(m), m \in \mathcal{M}_{\mathrm{RF}},
\end{array}
$$

with variables $\mathbf{V}, \mathbf{W}_{\mathrm{RF}}, \mathbf{W}_{\mathrm{BB}}, \mathbf{a}, \mathbf{c}, \mathbf{e}$; and $R$ is defined in (6).

\section{Algorithm Derivation}

Problem (5) is a nonconvex problem, and we propose a suboptimal algorithm to solve it. The proposed algorithm is based on the alternating optimization technique in conjunction with the sequential convex programming (SCP) [9], [10].

\section{A. Transmit Precoder Design}

The transmit analog/digital precoders are obtained by assuming that the optimal receiver structure (i.e., fixed $\mathbf{W}_{\mathrm{RF}}$, $\mathbf{W}_{\mathrm{BB}}$ ) is used. Furthermore, to efficiently find a solution we adopt a large-scale MIMO system assumption (where matrix $\mathbf{W}_{\mathrm{RF}}^{\mathrm{H}} \mathbf{W}_{\mathrm{RF}}$ is approximately a diagonal) [4], [11]. Then an approximate solution for problem (5) is obtained, by solving the following optimization problem:

$$
\begin{array}{ll}
\text { maximize } & B \log _{2}\left|\mathbf{I}+\frac{\left(1-\beta_{b}\right) \tilde{\mathbf{H}} \mathbf{V} \mathbf{V}^{\mathrm{H}} \tilde{\mathbf{H}}^{\mathrm{H}}}{\sigma^{2} \mathbf{\Upsilon}}\right| \\
\text { subject to } & \operatorname{tr}\left(\mathbf{V} \mathbf{V}^{\mathrm{H}}\right) \leq P_{\mathrm{tx}}^{\max },
\end{array}
$$


with variable $\mathbf{V}$, where $\tilde{\mathbf{H}}=\mathbf{W}_{\mathrm{RF}}^{\mathrm{H}} \mathbf{H}$ and $\boldsymbol{\Upsilon}=$ $\operatorname{diag}\left(\operatorname{diag}\left(\mathbf{W}_{\mathrm{RF}}^{\mathrm{H}} \mathbf{W}_{\mathrm{RF}}\right)\right)$. Note that in (7), constraints (5b)(5d) are dropped to find a fully-digital precoder $\mathbf{V}$.

Let $\left\{\overline{\mathbf{v}}_{1}, \ldots, \overline{\mathbf{v}}_{S}\right\}$ and $\left\{\alpha_{1}, \ldots, \alpha_{S}\right\}$ be the dominant right singular vectors and corresponding singular values of the matrix $\left(\left(1-\beta_{b}\right) / \sigma^{2}\right)^{1 / 2} \Upsilon^{-1 / 2} \tilde{\mathbf{H}}$. Then the fully-digital precoder for problem (7) can be expressed as [2, Ch. 7.1]

$$
\mathbf{V}^{\star}=\left[\sqrt{p}_{1} \overline{\mathbf{v}}_{1}, \ldots, \sqrt{p}_{S} \overline{\mathbf{v}}_{S}\right],
$$

where $\left\{p_{i}\right\}_{i=1, \ldots, S}$ are power allocation obtained by using water filling method, given by

$$
p_{i}=\left(\mu-1 / \alpha_{i}^{2}\right)^{+}, \quad i=1, \ldots, S,
$$

and a constant $\mu$ is chosen such that $\sum_{i=1}^{S} p_{i}=P_{\mathrm{tx}}^{\max }$.

1) Factorize $\mathbf{V}^{\star}$ into $\mathbf{V}_{\mathrm{RF}}$ and $\mathbf{V}_{\mathrm{BB}}$ : We derive alternating direction method of multipliers (ADMM) [12] based algorithm to factorize $\mathbf{V}^{\star}$ into $\mathbf{V}_{\mathrm{RF}}$ and $\mathbf{V}_{\mathrm{BB}}$. To do this, we minimize a Frobenius norm between $\mathbf{V}^{\star}$ and $\mathbf{V}_{\mathrm{RF}} \mathbf{V}_{\mathrm{BB}}$ subject to constraints (5c) and (5d) as follows:

$$
\begin{array}{ll}
\text { minimize } & \left\|\mathbf{V}^{\star}-\mathbf{V}_{\mathrm{RF}} \mathbf{V}_{\mathrm{BB}}\right\|_{F}^{2} \\
\text { subject to } & \left|\mathbf{V}_{\mathrm{RF}}(k, n)\right|^{2}=1, \quad k \in \mathcal{N}(n), n \in \mathcal{N}_{\mathrm{RF}} \\
& \mathbf{V}_{\mathrm{RF}}(k, n)=0, \quad k \in \overline{\mathcal{N}}(n), n \in \mathcal{N}_{\mathrm{RF}},
\end{array}
$$

with variables $\mathbf{V}_{\mathrm{RF}}$ and $\mathbf{V}_{\mathrm{BB}}$.

Note that problem (10) is not a convex optimization problem, and ADMM is not guaranteed to converge for a nonconvex problem [12]. However, if each step of the ADMM iteration is tractable, the ADMM algorithm can still be used to derive (possibly suboptimal) solution method for nonconvex problem [12, Ch. 9]. To derive ADMM algorithm for problem (10), we start by introducing an auxiliary variable $\mathbf{Z}$ as a copy of $\mathbf{V}_{\mathrm{RF}}$. Then problem (10) can be equivalently written as

$$
\begin{array}{ll}
\text { minimize } & \left\|\mathbf{V}^{\star}-\mathbf{Z} \mathbf{V}_{\mathrm{BB}}\right\|_{F}^{2} \\
\text { subject to } & \mathbf{Z}=\mathbf{V}_{\mathrm{RF}} \\
& \left|\mathbf{V}_{\mathrm{RF}}(k, n)\right|^{2}=1, k \in \mathcal{N}(n), n \in \mathcal{N}_{\mathrm{RF}} \\
& \mathbf{V}_{\mathrm{RF}}(k, n)=0, k \in \mathcal{N}(n), n \in \mathcal{N}_{\mathrm{RF}},
\end{array}
$$

with variables $\mathbf{Z}, \mathbf{V}_{\mathrm{RF}}$, and $\mathbf{V}_{\mathrm{BB}}$.

For convenience, we express problem (11) compactly. To do this we introduce a set $\mathcal{V}_{\mathrm{RF}}$ and function $g\left(\mathbf{V}_{\mathrm{RF}}\right)$ as

$$
\begin{aligned}
\mathcal{V}_{\mathrm{RF}} & =\left\{\mathbf{V}_{\mathrm{RF}} \mid \text { constraints }(5 \mathrm{c}) \text { and }(5 \mathrm{~d})\right\}, \\
g\left(\mathbf{V}_{\mathrm{RF}}\right) & = \begin{cases}0 & \mathbf{V}_{\mathrm{RF}} \in \mathcal{V}_{\mathrm{RF}} \\
\infty & \text { otherwise }\end{cases}
\end{aligned}
$$

Then problem (11) can be written compactly as

$$
\begin{array}{ll}
\text { minimize } & \left\|\mathbf{V}^{\star}-\mathbf{Z} \mathbf{V}_{\mathrm{BB}}\right\|_{F}^{2}+g\left(\mathbf{V}_{\mathrm{RF}}\right) \\
\text { subject to } & \mathbf{Z}=\mathbf{V}_{\mathrm{RF}},
\end{array}
$$

with variables $\mathbf{Z}, \mathbf{V}_{\mathrm{RF}}$, and $\mathbf{V}_{\mathrm{BB}}$.

ADMM algorithm: To derive the ADMM algorithm we first form the augmented Lagrangian [12] of problem (14). Let $\Omega \in \mathbb{C}^{N_{\mathrm{RF}} \times N}$ be the dual variable associated with the equality constraint of problem (14). Then the augmented Lagrangian for problem (14), with the equality constraints on the complex variables, can be written as [13]

$$
\begin{array}{r}
L_{\rho}\left(\mathbf{Z}, \mathbf{V}_{\mathrm{BB}}, \mathbf{V}_{\mathrm{RF}}, \boldsymbol{\Psi}\right)=\left\|\mathbf{V}^{\star}-\mathbf{Z} \mathbf{V}_{\mathrm{BB}}\right\|_{F}^{2}+g\left(\mathbf{V}_{\mathrm{RF}}\right) \\
+\rho\left\|\mathbf{Z}-\mathbf{V}_{\mathrm{RF}}+\boldsymbol{\Psi}\right\|_{F}^{2}-\rho\|\boldsymbol{\Psi}\|_{F}^{2} .
\end{array}
$$

where $\rho>0$ and $\boldsymbol{\Psi}=(1 / \rho) \boldsymbol{\Omega}$.

To obtain the ADMM iteration, let $\mathbf{Z}$ be the first block, and $\left(\mathbf{V}_{\mathrm{BB}}, \mathbf{V}_{\mathrm{RF}}\right)$ be the second block. Then each iteration of ADMM algorithm consists of the following three steps [12]:

$$
\begin{aligned}
\mathbf{Z}^{l+1} & =\underset{\mathbf{Z}}{\operatorname{argmin}} L_{\rho}\left(\mathbf{Z}, \mathbf{V}_{\mathrm{BB}}^{l}, \mathbf{V}_{\mathrm{RF}}^{l}, \Psi^{l}\right) \\
\left(\mathbf{V}_{\mathrm{BB}}^{l+1}, \mathbf{V}_{\mathrm{RF}}^{l+1}\right) & =\underset{\mathbf{V}_{\mathrm{BB}}, \mathbf{V}_{\mathrm{RF}} \in \mathcal{V}_{\mathrm{RF}}}{\operatorname{argmin}} L_{\rho}\left(\mathbf{Z}^{l+1}, \mathbf{V}_{\mathrm{BB}}, \mathbf{V}_{\mathrm{RF}}, \Psi^{l}\right) \\
\Psi^{l+1} & =\mathbf{\Psi}^{l}+\mathbf{Z}^{l+1}-\mathbf{V}_{\mathrm{RF}}^{l+1},
\end{aligned}
$$

where $l$ is the iteration counter. It is easy to see that expression (17) is separable in variables $\mathbf{V}_{\mathrm{BB}}$ and $\mathbf{V}_{\mathrm{RF}}$. Furthermore, updates $\mathbf{Z}^{l+1}$ and $\mathbf{V}_{\mathrm{BB}}^{l+1}$ are solutions of unconstrained quadratic optimization problems. Thus, we can get

$$
\begin{aligned}
\mathbf{Z}^{l+1} & =\left(\mathbf{V}^{\star} \mathbf{V}_{\mathrm{BB}}^{l \mathrm{H}}+\rho \mathbf{V}_{\mathrm{RF}}^{l}-\rho \mathbf{\Psi}^{l}\right)\left(\mathbf{V}_{\mathrm{BB}}^{l} \mathbf{V}_{\mathrm{BB}}^{l \mathrm{H}}+\rho \mathbf{I}\right)^{-1}(19) \\
\mathbf{V}_{\mathrm{BB}}^{l+1} & =\left(\mathbf{Z}^{(l+1) \mathrm{H}} \mathbf{Z}^{l+1}\right)^{-1} \mathbf{Z}^{(l+1) \mathrm{H}} \mathbf{V}^{\star} .
\end{aligned}
$$

The update $\mathbf{V}_{\mathrm{RF}}^{l+1}$ in expression (17) can be obtained by solving the following optimization problem:

$$
\begin{array}{ll}
\text { minimize } & \left\|\Psi^{l}+\mathbf{Z}^{l+1}-\mathbf{V}_{\mathrm{RF}}\right\|_{F}^{2} \\
\text { subject to } & \left|\mathbf{V}_{\mathrm{RF}}(k, n)\right|^{2}=1, k \in \mathcal{N}(n), n \in \mathcal{N}_{\mathrm{RF}} \\
& \mathbf{V}_{\mathrm{RF}}(k, n)=0, k \in \overline{\mathcal{N}}(n), n \in \mathcal{N}_{\mathrm{RF}},
\end{array}
$$

Problem (21) is separable in each variable $\mathbf{V}_{\mathrm{RF}}(k, n)$. Thus a solution $\mathbf{V}_{\mathrm{RF}}^{\star}(k, n)$ can be expressed as

$$
\mathbf{V}_{\mathrm{RF}}^{\star}(k, n)=e^{j \theta} \text {, with } \theta=\arg \left(\Psi^{l}(k, n)+\mathbf{Z}^{l+1}(k, n)\right),
$$

for all $k \in \mathcal{N}$ and $n \in \mathcal{N}_{\mathrm{RF}}$.

\section{B. Analog Combiner Design}

In order to obtain analog combiner $\mathbf{W}_{\mathrm{RF}}$ and the associated components (i.e., the required number of RF chains, PSs, and LNAs) transmit analog/digital precoders $\left(\mathbf{V}_{\mathrm{RF}}, \mathbf{V}_{\mathrm{BB}}\right)$ are fixed in problem (5). Furthermore, the $\operatorname{diag}(\cdot)$ operator is relaxed, and problem (5) is approximated as

$$
\begin{aligned}
\operatorname{maximize} & f_{0}\left(\mathbf{D}, \mathbf{W}_{\mathrm{RF}}\right) \\
\text { subject to } & \text { constraints }(5 \mathrm{e})-(51) \\
& \mathbf{D}=\mathbf{I}-\operatorname{diag}\left(c_{1}, \ldots, c_{M_{\mathrm{RF}}}\right),
\end{aligned}
$$

with variables $\mathbf{W}_{\mathrm{RF}}, \mathbf{a}, \mathbf{c}, \mathbf{e}$, and $\mathbf{D}$. In problem (22) function $f_{0}\left(\mathbf{D}, \mathbf{W}_{\mathrm{RF}}\right)$ is defined as

$$
f_{0}\left(\mathbf{D}, \mathbf{W}_{\mathrm{RF}}\right)=B \log _{2} \frac{\left|\mathbf{D}+\mathbf{W}_{\mathrm{RF}}^{\mathrm{H}}\left(\sigma^{2} \mathbf{I}+\mathbf{G}\right) \mathbf{W}_{\mathrm{RF}}\right|}{\left|\mathbf{D}+\mathbf{W}_{\mathrm{RF}}^{\mathrm{H}}\left(\sigma^{2} \mathbf{I}+\beta_{b} \mathbf{G}\right) \mathbf{W}_{\mathrm{RF}}\right|},
$$

where $\mathbf{G}=\mathbf{H V V}^{\mathrm{H}} \mathbf{H}^{\mathrm{H}}$. Note that the use of auxiliary variable $\mathbf{D}$ makes the term $\left|\mathbf{D}+\mathbf{W}_{\mathrm{RF}}^{\mathrm{H}}\left(\sigma^{2} \mathbf{I}+\beta_{b} \mathbf{G}\right) \mathbf{W}_{\mathrm{RF}}\right|$ nonsingular. However, it does not affect the objective of problem (5). 
In function $f_{0}\left(\mathbf{D}, \mathbf{W}_{\mathrm{RF}}\right)$, we can see that if numerator $\mid \mathbf{D}+$ $\mathbf{W}_{\mathrm{RF}}^{\mathrm{H}}\left(\sigma^{2} \mathbf{I}+\mathbf{G}\right) \mathbf{W}_{\mathrm{RF}} \mid$ increases, the term in denominator $\mid \mathbf{D}+$ $\mathbf{W}_{\mathrm{RF}}^{\mathrm{H}}\left(\sigma^{2} \mathbf{I}+\beta_{b} \mathbf{G}\right) \mathbf{W}_{\mathrm{RF}} \mid$ also increases. We further exploit this relative incremental behavior in function $f_{0}\left(\mathbf{D}, \mathbf{W}_{\mathrm{RF}}\right)$, and find an approximate solution for problem (22) by solving the following optimization problem:

$$
\begin{aligned}
\operatorname{maximize} & B \log _{2}\left|\mathbf{D}+\mathbf{W}_{\mathrm{RF}}^{\mathrm{H}}\left(\sigma^{2} \mathbf{I}+\mathbf{G}\right) \mathbf{W}_{\mathrm{RF}}\right| \\
& +\delta \sum_{m \in \mathcal{M}_{\mathrm{RF}}} \sum_{k \in \mathcal{M}(m)} a_{k m} \log \left(a_{k m}\right) \\
\text { subject to } & P_{\mathrm{rx}}(\mathbf{a}, b, \mathbf{c}, \mathbf{e}) \leq P_{\mathrm{rx}}^{\max } \\
& \left|\mathbf{W}_{\mathrm{RF}}(k, m)\right|^{2} \leq a_{k m}, \quad k \in \mathcal{M}, m \in \mathcal{M}_{\mathrm{RF}} \\
& a_{k m} \leq c_{m}, \quad k \in \mathcal{M}(m), m \in \mathcal{M}_{\mathrm{RF}} \\
& a_{k m} \leq e_{k}, \quad m \in \mathcal{M}_{\mathrm{RF}}, k \in \mathcal{M} \\
& 0 \leq a_{k m} \leq 1, \quad k \in \mathcal{M}(m), m \in \mathcal{M}_{\mathrm{RF}} \\
& a_{k m}=0, \quad k \in \overline{\mathcal{M}}(m), m \in \mathcal{M}_{\mathrm{RF}} \\
& 0 \leq c_{m} \leq 1, \quad m \in \mathcal{M} \mathrm{RF} \\
& 0 \leq e_{k} \leq 1, \quad k \in \mathcal{M} \\
& \mathbf{D}=\mathbf{I}-\operatorname{diag}\left(c_{1}, \ldots, c_{M_{\mathrm{RF}}}\right)
\end{aligned}
$$

with variables $\mathbf{W}_{\mathrm{RF}}$, a, c, e, and $\mathbf{D}$; a parameter $\delta>0$. Note that we have relaxed the binary constraints in (22a), and employed a penalty function $a_{k m} \log \left(a_{k m}\right)$ (negative entropy function) to promote binary values for $\left\{a_{k m}\right\}$. Function $a_{k m} \log \left(a_{k m}\right)$ has maximum values at $a_{k m}$ equal to zero or one. Thus, there exits a value of parameter $\delta$ that can achieve binary values for variables $\left\{a_{k m}\right\}$.

Problem (23) is still not a convex, and we use the SCP approach [10] to solve it. By using the first order approximation [14, App. A.4] of the objective function near arbitrary points $\hat{\mathbf{D}}, \hat{\mathbf{W}}_{\mathrm{RF}}$, and $\left\{\hat{a}_{k m}\right\}_{k \in \mathcal{M}(m), m \in \mathcal{M}_{\mathrm{RF}}}$, a convex approximation of problem (23) can be written as

$$
\begin{aligned}
\operatorname{maximize} & B \operatorname{tr}\left(\left(\hat{\mathbf{D}}+\hat{\mathbf{W}}_{\mathrm{RF}}^{\mathrm{H}} \mathbf{F} \hat{\mathbf{W}}_{\mathrm{RF}}\right)^{-1}\left(\mathbf{D}+2 \hat{\mathbf{W}}_{\mathrm{RF}}^{\mathrm{H}} \mathbf{F} \mathbf{W}_{\mathrm{RF}}\right)\right) \\
& +\delta \sum_{m \in \mathcal{M}_{\mathrm{RF}}} \sum_{k \in \mathcal{M}(m)}\left(1+\log \left(\hat{a}_{k m}\right)\right) a_{k m}
\end{aligned}
$$

subject to constraints (23a) $-(23 h)$,

with variables $\mathbf{W}_{\mathrm{RF}}, \mathbf{a}, \mathbf{c}, \mathbf{e}$, and $\mathbf{D}$; where $\mathbf{F}=\sigma^{2} \mathbf{I}+\mathbf{G}$.

Problem (24) approximates a solution for (23) near arbitrary points $\hat{\mathbf{D}}, \hat{\mathbf{W}}_{\mathrm{RF}}$, and $\left\{\hat{a}_{k m}\right\}$. Hence, to obtain the best local solution for (23), we need to solve (24) repeatedly for different values of $\hat{\mathbf{D}}, \hat{\mathbf{W}}_{\mathrm{RF}}$, and $\left\{\hat{a}_{k m}\right\}$. Thus, we take the solution $\mathbf{D}^{\star}, \mathbf{W}_{\mathrm{RF}}^{\star}$ and $\mathbf{a}^{\star}$ of problem (24) as the next iterate (i.e., we set $\hat{\mathbf{D}}=\mathbf{D}^{\star}, \hat{\mathbf{W}}_{\mathrm{RF}}=\mathbf{W}_{\mathrm{RF}}^{\star}$, and $\left\{\hat{a}_{k m}=\right.$

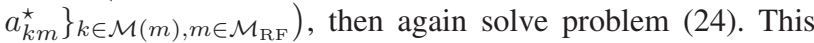
step is repeated until convergence.

\section{Digital Baseband Combiner Design}

For fixed transmitter and analog combiner, we obtain MMSE solution for $\mathbf{W}_{\mathrm{BB}}$, and it can be expressed as [15]

$$
\mathbf{W}_{\mathrm{BB}}=\mathbf{J}^{-1} \mathbf{W}_{\mathrm{RF}}^{\mathrm{H}} \mathbf{H} \mathbf{V}_{\mathrm{RF}} \mathbf{V}_{\mathrm{BB}},
$$

where $\mathbf{J}$ is given by

$$
\begin{aligned}
\mathbf{J}= & \left(1-\beta_{b}\right) \mathbf{W}_{\mathrm{RF}}^{\mathrm{H}} \mathbf{H} \mathbf{V} \mathbf{V}^{\mathrm{H}} \mathbf{H}^{\mathrm{H}} \mathbf{W}_{\mathrm{RF}}+\sigma^{2}\left(1-\beta_{b}\right) \mathbf{W}_{\mathrm{RF}}^{\mathrm{H}} \mathbf{W}_{\mathrm{RF}} \\
& +\beta_{b} \operatorname{diag}\left(\mathbf{W}_{\mathrm{RF}}^{\mathrm{H}} \mathbf{H V} \mathbf{V}^{\mathrm{H}} \mathbf{H}^{\mathrm{H}} \mathbf{W}_{\mathrm{RF}}+\sigma^{\mathbf{2}} \mathbf{W}_{\mathrm{RF}}^{\mathrm{H}} \mathbf{W}_{\mathrm{RF}}\right) .
\end{aligned}
$$

Finally, we summarized a proposed algorithm to solve problem (5) below.

Algorithm 1: Design hybrid beamforming for problem (5).

1) Initialization: feasible points $\mathbf{D}^{0}, \mathbf{W}_{\mathrm{RF}}^{0}$, and $\left\{a_{k m}^{0}\right\}$; parameter $\delta>0$. Obtain singular value decomposition of $\mathbf{H}=\hat{\mathbf{U}} \boldsymbol{\Sigma} \hat{\mathbf{V}}^{\mathrm{H}}$, and set iteration index $i=0$.

2) Receiver analog components design:

a) Set $\tilde{\mathbf{V}}=\left(P_{\mathrm{tx}}^{\max } / S\right) \hat{\mathbf{V}}$, and $\mathbf{G}=\mathbf{H} \tilde{\mathbf{V}} \tilde{\mathbf{V}}^{\mathrm{H}} \mathbf{H}^{\mathrm{H}}$.

b) Set $\hat{\mathbf{D}}=\mathbf{D}^{i}, \hat{\mathbf{W}}_{\mathrm{RF}}=\mathbf{W}_{\mathrm{RF}}^{i}$ and $\left\{\hat{a}_{k m}=\right.$ $\left.a_{k m}^{i}\right\}_{k \in \mathcal{M}(m), m \in \mathcal{M}_{\mathrm{RF}}}$.

c) Solve problem (24), and denote the solution by $\left\{\mathbf{W}_{\mathrm{RF}}^{\star}, \mathbf{a}^{\star}, \mathbf{c}^{\star}, \mathbf{e}^{\star}, \mathbf{D}^{\star}\right\}$. Set $\mathbf{D}^{i+1}=\mathbf{D}^{\star}, \mathbf{W}_{\mathrm{RF}}^{i+1}=$ $\mathbf{W}_{\mathrm{RF}}^{\star}$ and $\left\{a_{k m}^{i+1}=a_{k m}^{\star}\right\}_{k \in \mathcal{M}(m), m \in \mathcal{M}_{\mathrm{RF}}}$.

d) Stopping criteria: if stopping criteria is satisfied, STOP. Otherwise set $i=i+1$, and go to step $2 \mathrm{~b}$.

3) Obtain transmit precoder $V^{\star}$ by using expression (8).

4) Factorize $V^{\star}$ into $V_{R F}$ and $V_{B B}$ by ADMM iterations (16)-(18).

5) Obtain digital combiner $\mathbf{W}_{\mathrm{BB}}$ by using expression (25).

\section{Simulation RESUlts}

The hybrid transceiver architecture as shown in Fig. 1(a) is considered. We assume that the transmitter and receiver are equipped with $N=64$ and $M=8$ antennas, respectively, and they communicate via $S=3$ data streams. We set $N_{\mathrm{RF}}=6 \mathrm{RF}$ chains at the transmitter. A geometric path loss model [4], [5] with $L$ scattering clusters is considered. Furthermore, we assume a uniform linear array antenna configuration. Thus, a channel $\mathbf{H} \in \mathbb{C}^{M \times N}$ can be modeled as $\mathbf{H}=\sqrt{M N / L} \sum_{l=1}^{L} \alpha_{l} \mathbf{s}_{\mathrm{rx}}\left(\theta_{l}\right) \mathbf{s}_{\mathrm{tx}}\left(\phi_{l}\right)^{\mathrm{H}}$, where $\alpha_{l}$ is a complex gain of the $l$ th channel tap, and vectors $\mathbf{s}_{\mathrm{rx}}\left(\theta_{l}\right)$ and $\mathbf{s}_{\mathrm{tx}}\left(\phi_{l}\right)$ are expressed as

$$
\begin{aligned}
\mathbf{s}_{\mathrm{rx}}\left(\theta_{l}\right. & =(1 / \sqrt{M})\left[1, e^{j \omega d \sin \left(\theta_{l}\right)}, \ldots, e^{j \omega d(M-1) \sin \left(\theta_{l}\right)}\right]^{\mathrm{T}} \\
\mathbf{s}_{\mathrm{tx}}\left(\phi_{l}\right) & =(1 / \sqrt{N})\left[1, e^{j \omega d \sin \left(\phi_{l}\right)}, \ldots, e^{j \omega d(N-1) \sin \left(\phi_{l}\right)}\right]^{\mathrm{T}},
\end{aligned}
$$

where $\omega=2 \pi / \lambda, \lambda$ is wavelength, and $d$ is antenna spacing. In the simulation, we set $L=6, d=\lambda / 2, \alpha_{l} \sim \mathcal{C N}(0,1)$, $\theta_{l} \in[0,2 \pi)$, and $\phi_{l} \in[0,2 \pi)$.

The exact computation of the dissipated power by each component of receiver is difficult. To model receiver power consumption, we assume $P_{\mathrm{LNA}}=20 \mathrm{~mW}$, $P_{\mathrm{PS}}=10 \mathrm{~mW}, P_{\mathrm{RF} \text { chain }}=40 \mathrm{~mW}, P_{\mathrm{BB}}=200 \mathrm{~mW}$, $\xi=500 \mathrm{fJ} /$ conversion-step, and channel bandwidth $B=$ $1 \mathrm{GHz}[16]-[18]$.

Fig. 2 shows spectral efficiency versus ADC resolution $b$ for different values of receiver power constraint $P_{\mathrm{rx}}^{\max }$. As a benchmark fully-digital MIMO with infinite resolution ADC 
is considered. Results show that for small values of $P_{\mathrm{rx}}^{\max }$ (i.e., power-constrained receiver), the achievable rate decreases with increase in the ADC resolution. It is because ADC power consumption scales exponentially with bits $b$ per sample [8], and there is not sufficient power to activate enough RF chains. This we can see in Fig. 3.

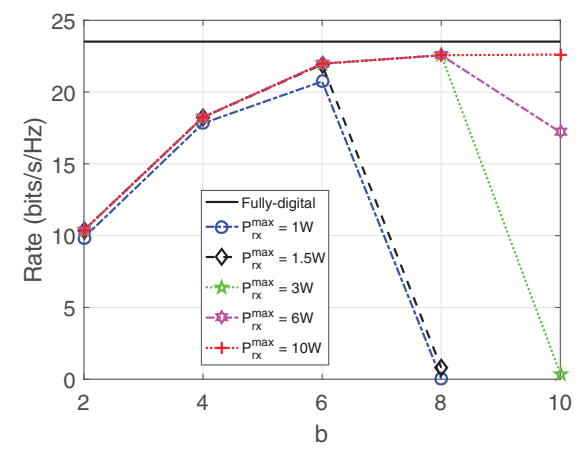

Fig. 2. Average rate versus $\mathrm{ADC}$ resolution for $\mathrm{SNR}=0 \mathrm{~dB}$.

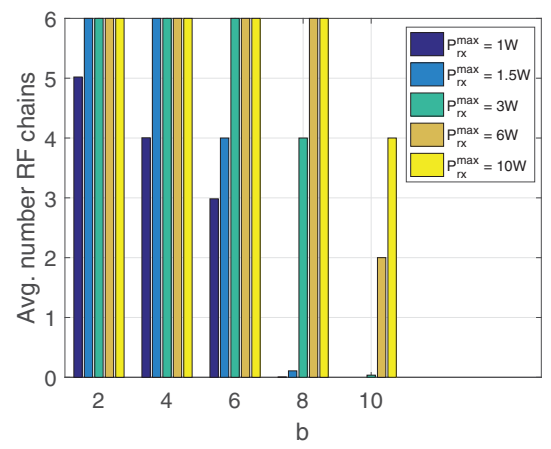

Fig. 3. Average number of active RF chain versus ADC resolution for $\mathrm{SNR}=0 \mathrm{~dB}$

Fig. (4) shows the spectral efficiency versus SNR for $P_{\mathrm{rx}}^{\max }=3 \mathrm{~W}$. Plots are drawn for ADC resolution $b=$ $2,4,6,8,10$ bits and for the fully-digital MIMO system. Results show that the achievable rate approach the fully-digital MIMO system with the increase in ADC resolution $b$, only if the receiver is not power-limited. For example, in Fig. 4 when ADC $b=10$ bit, the achievable rate is smaller than that for $b=2$ bits. This is because $P_{\mathrm{rx}}^{\max }=3 \mathrm{~W}$ is not enough to support power consuming high-precision ADCs.

\section{CONCLUSIONS}

We have considered the problem of hybrid beamforming with low resolution ADC for millimeter wave communications. The hybrid precoder/decoder design algorithm for maximizing the spectral efficiency has been proposed. Numerical results show that there is a trade-off between the number of active RF chains and ADC resolution, for power constrained hybrid receiver, that can obtain maximal spectral efficiency.

\section{REFERENCES}

[1] T. S. Rappaport, S. Sun, R. Mayzus, H. Zhao, Y. Azar, K. Wang, G. N. Wong, J. K. Schulz, M. Samimi, and F. Gutierrez, "Millimeter wave

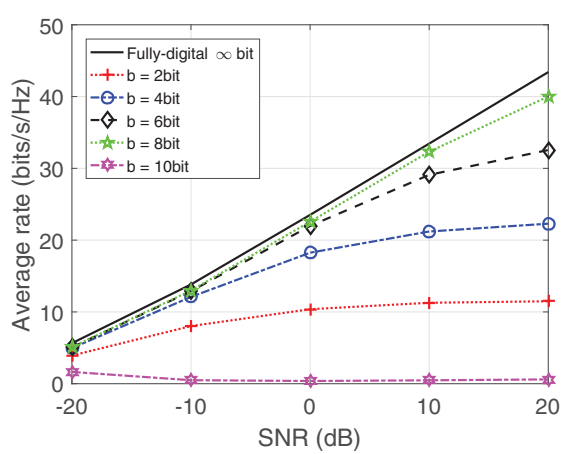

Fig. 4. Average rate versus SNR for $P_{\mathrm{rx}}^{\max }=3 \mathrm{~W}$.

mobile communications for 5G cellular: It will work!" IEEE Access, vol. 1, pp. 335-349, May 2013.

[2] D. Tse and P. Viswanath, Fundamentals of Wireless Communication. Cambridge, UK: Cambridge University Press, 2005.

[3] E. Björnson and E. Jorswieck, "Optimal resource allocation in coordinated multi-cell systems," Found. Trends Commun. Inform. Th., vol. 9, no. 2-3, pp. 111-381, Jan. 2013.

[4] F. Sohrabi and W. Yu, "Hybrid digital and analog beamforming design for large-scale antenna arrays," IEEE J. Select. Topics Signal Processing, vol. 10, no. 3, pp. 501-513, Apr. 2016.

[5] L. Liang, W. Xu, and X. Dong, "Low-complexity hybrid precoding in massive multiuser MIMO systems," IEEE Commun. Lett., vol. 3, no. 6, pp. 653-656, Dec. 2014.

[6] A. Mezghani and J. Nossek, "Capacity lower bound of MIMO channels with output quantization and correlated noise," in Proc. IEEE Int. Symp. Inform. Theory, Cambridge, MA, Jul. 2012.

[7] J. Max, "Quantizing for minimum distortion," IRE Trans. Inform. Theory, vol. 6, pp. 7-12, 1960.

[8] H.-S. Lee and C. G. Sodini, "Analog-to-digital converters: Digitizing the analog world," Proc. IEEE, vol. 96, no. 2, pp. 323-334, Feb. 2008.

[9] U. Niesen, D. Shah, and G. W. Wornell, "Adaptive alternating minimization algorithms," IEEE Trans. Inform. Theory, vol. 55, no. 3, pp. 1423-1429, Mar. 2009.

[10] S. Boyd, "Sequential convex programming," 2007, [Online]. Available: http://www.stanford.edu/class/ee364b/lectures/seq_slides.pdf.

[11] O. E. Ayach, S. Rajagopal, S. Abu-Surra, Z. Pi, and R. W. Heath, "Spatially sparse precoding in millimeter wave MIMO systems," IEEE Trans. Wireless Commun., vol. 13, no. 3, pp. 1499-1513, Mar. 2014.

[12] S. Boyd, N. Parikh, E. Chu, B. Peleato, and J. Eckstein, "Distributed optimization and statistical learning via the alternating direction method of multipliers," Found. Trends Mach. Learn., vol. 3, no. 1, pp. 1-122, 2010.

[13] L. Li, X. Wang, and G. Wang, "Alternating direction method of multipliers for separable convex optimization of real functions in complex variables," Hindawi, Math. Prob. Eng., vol. 2015, 2015.

[14] S. Boyd and L. Vandenberghe, Convex Optimization. Cambridge, UK: Cambridge University Press, 2004.

[15] D. P. Palomar, "A unified framework for communications through MIMO channels," Ph.D. dissertation, Department of Signal Theory and Communications, Technical University of Catalonia, Barcelona, Spain, May 2003.

[16] R. M.-Rial, C. Rusu, N. G.-Prelcic, A. Alkhateeb, and R. W. Heath, "Hybrid MIMO architectures for millimeter wave communications: Phase shifters or switches?" IEEE Access, vol. 4, pp. 247-267, Jan. 2016.

[17] Y.-H. Lin and H. Wang, "A low phase and gain error passive phase shifter in $90 \mathrm{~nm}$ CMOS for $60 \mathrm{GHz}$ phase array system application," in IEEE Int. Microwave Symposium, May 2016, pp. 1-4.

[18] A. M. A. Ali and et al, "A 14 bit 1 GS/s RF sampling pipelined ADC with background calibration," IEEE Journal of Solid-State Circuits, vol. 49, no. 12, pp. 2857-2867, Dec. 2014. 